\title{
A Graph-Theoretic Method to Representing a Concept Lattice
}

\author{
Hua Mao* \\ Department of Mathematics, Hebei University, Baoding 071002, China
}

Received: 17 Mar. 2013, Revised: 18 Jul. 2013, Accepted: 20 Jul. 2013

Published online: 1 Mar. 2014

\begin{abstract}
Concept lattices are indeed lattices. In this paper, we present a new relationship between lattices and graphs: given a binary relation $I$, we define an underlying graph $D_{I}$, and find out the constitution in the set of cover elements of the minimum element of the concept lattice of $I$ using the properties of $D_{I}$. The following is to provide a way to establish a one-to-one correspondence between the set of covers of an element in the concept lattice and the set of covers of the minimum in a sublattice of the concept lattice. We apply the one-to-one correspondence to define a new underlying graph, and generate the elements of the lattice.
\end{abstract}

Keywords: concept lattice; graph; neighbor; cover; interval

\section{Introduction and Preliminaries}

We know that many problems of data analysis are naturally formulated in terms of formal concept lattice. As A.Berry and A.Sigayret said in [4], one of the important challenges in data handling is generating or navigating the concept lattice of a binary relation.

In this paper, we provide a graph-theoretic approach by determining the concept lattice of a binary relation with the underlying graph. An important step in connecting this graph-theoretic approach with lattices is to associate each binary relation with an underlying undirected bipartite graph. When the bipartite graph is obtained, for the minimum element in the concept lattice of the binary relation, all its cover elements are searched out by the graph-theoretic method. Additionally, a key aspect of this approach is that it equates the concepts of the lattice with the minimum element in a new binary relation.

In summary, our method presented in this paper is an initial structural results, which we expect will provide support for further advance in this direction.

The outline of the paper is as follows. After introducing some notions from concept lattice, graph theory and lattice theory, we define the underlying graph $D_{I}$ which we use to represent a binary relation $I$, and describe some of its properties. Then, we present the main results regarding the method of obtaining the concept lattice of $I$ in this paper. This is followed by a description of the manner in which the concept lattice can be computed and visualized in steps, at the same time, the diagram of the concept lattice is born. The final section gives an example for illustrating the graph-theoretic method presented in this paper.

Although some of the definitions appearing in this section do not require that the sets involved be finite, we make a standing assumption that all the discussions under consideration are finite. Originally, the terminologies of concept lattices are given below. After that, some known properties about concept lattices are shown.

Definition 1 [1,3] A triple $(G, M, I)$ is called a formal context, if $G$ and $M$ are sets and $I \subseteq G \times M$ is a binary relation between $G$ and $M$. We call the elements of $G$ objects, those of $M$ attributes, and $I$ the incidence of $(G, M, I)$. For $A \subseteq G$, we define

$A^{\prime}=\{m \in M \mid(g, m) \in I$, for all $g \in A\}$, and dually, for $B \subseteq M$

$B^{\prime}=\{g \in G \mid(g, m) \in I$, for all $m \in B\}$.

$(A, B)$ is a formal concept of $(G, M, I)$ if and only if $A \subseteq G, B \subseteq M, A^{\prime}=B$ and $A=B^{\prime}$.

The concepts of a given context are naturally ordered by the relation defined by

$\left(A_{1}, B_{1}\right) \leq\left(A_{2}, B_{2}\right) \Leftrightarrow A_{1} \subseteq A_{2}\left(\Leftrightarrow B_{2} \subseteq B_{1}\right)$.

\footnotetext{
*Corresponding author e-mail: yushengmao@263.net
} 
The ordered set of all formal concepts of $(G, M, I)$ is denoted by $\mathscr{B}(G, M, I)$ and it is called the concept lattice of $(G, M, I)$.

In this paper, a formal context and a formal concept will be simply said a context and a concept respectively; $(g, m) \in I$ is often written as $g I m$.

Lemma $1[1,3]$ (1) For $A_{1}, A_{2}, A \subseteq G$ and $B_{1}, B_{2}, B \subseteq$ $M$, there are the following statements:

(i) $A_{1} \subseteq A_{2} \Rightarrow A_{2}^{\prime} \subseteq A_{1}^{\prime}$. (i)' $B_{1} \subseteq B_{2} \Rightarrow B_{2}^{\prime} \subseteq B_{1}^{\prime}$.

(ii) $A \subseteq A^{\prime \prime}$ and $A^{\prime}=A^{\prime \prime \prime}$. (ii)' $B \subseteq B^{\prime \prime}$ and $B^{\prime}=B^{\prime \prime \prime}$.

(iii) $A \subseteq B^{\prime} \Leftrightarrow B \subseteq A^{\prime}$.

(2) The concept lattice $\mathscr{B}(G, M, I)$ is complete.

The graphs used here are finite and undirected. About graph theory's knowledge, we just show some of them next and the others are referred to [2]. A graph $D=(V(D), E(D))$ means that $V(D)$ is the vertex set and $E(D) \subseteq V(D)^{2}=\{x y \mid x, y \in V(D)\}$ is the edge set.

Definition 2 [2] A bipartite graph is one whose vertex set can be partitioned into two subsets $X$ and $Y$, so that each edge has one end in $X$ and one end in $Y$.

The degree $d_{D}(u)$ of $u \in V(D)$ is the number of edges of $D$ incident with $u$.

Two vertices $u$ and $v$ of $D$ are said to be connected if there is a $(u, v)$-path in $D$. Connection is an equivalence relation on the vertex set $V$. Thus, there is a partition of $V$ into nonempty subsets $V_{1}, V_{2}, \ldots, V_{w}$ such that two vertices $u$ and $v$ are connected if and only if both $u$ and $v$ belong to the same set $V_{i}$.

The subgraphs $D\left[V_{1}\right], D\left[V_{2}\right], \ldots, D\left[V_{w}\right]$ are called the components of $D$. If $D$ has exactly one component, $G$ is connected; otherwise, $D$ is disconnected.

For any set $S$ of vertices in $D$, we define the neighbor set of $S$ in $D$ to be the set of all vertices adjacent to vertices in $S$; this set is denoted by $N_{D}(S)$.

We will use $d(u)$ and $N(S)$ instead of $d_{D}(u)$ and $N_{D}(S)$ respectively if it does not cause confusion throughout the rest of the paper.

By Lemma 1, we see that $\mathscr{B}(G, M, I)$ is indeed a lattice. We know that the main goal of data analysis is just to find the lattice construction for a given context. Hence, we also need lattice theory to finish this duty. We just write out some known literature about lattice theory, the others of lattice theory are seen [3].

Definition 3 [3] The diagram of a poset $(P, \leq)$ represents the elements with small circles; the circle representing two elements $x, y$ are connected by a straight line if and only if one covers the other; if $x$ covers $y$, then the circle representing $x$ is higher than the circle representing $y$.

Let $L$ be a lattice. For $a, b \in L$ and $a \leq b$, the interval $[a, b]=\{x \in L \mid a \leq x \leq b\}$.
In view of the results in [3], we know that $[a, b]$ is a sublattice of $L$.

\section{The bipartite graph underlying a binary relation}

In the previous works, [4] introduces to represent a given context by a graph constructed on the complement of the relation; the breath first search graph partitions method is shown in [5]. To benefit by the ideas in [4,5], we just construct a graph on a given context to obtain the concept lattice and the diagram of the concept lattice. Of course, our graph is quite different from that in $[4,5]$ and the other materials such as the references in $[4,5]$. Thus the method here is a new approach.

We should point out that the relations we work on are considered as non-empty.

Definition 4 Let $(G, M, I)$ be a context; we will define an associated underlying graph, denoted $D_{I}$, as follows:

- The vertex set of $D_{I}$ is $G \cup M$.

- For $x, y \in G$, there is not any edge to incident with $x$ and $y$.

- For $x, y \in M$, there is not any edge to incident with $x$ and $y$.

- For a vertex $x$ of $G$ and a vertex $y$ of $M$, there is an edge in $D_{I}$ if and only if $(x, y)$ is in $I$.

Note that only the vertices between a vertex of $G$ and a vertex of $M$ are possible to be incident with an edge; the vertices between $G$ or between $M$ are not possible to be incident with an edge. Thus, $D_{I}$ is a bipartite graph and undirected graph and $V\left(D_{I}\right)=G \cup M$ and $E\left(D_{I}\right)=\{x y \mid$ $x \in G, y \in M,(x, y) \in I\}=\{y x \mid y \in M, x \in G,(x, y) \in I\}$. The graphs of this class have several remarkable properties, such as hereditary: any subgraph of bipartite graph which has more than one vertex is again bipartite graph. Moreover, since the relations we work on are considered as non-empty, $D_{I}$ is always hereditary.

We present several nice properties on $D_{I}$, which makes our construction $\mathscr{B}(G, M, I)$ easier to handle than on more general graphs.

Lemma 2 Let $D_{1}, D_{2}, \ldots, D_{\gamma}$ be all the components of $D_{I}$ associated with $\mathscr{B}(G, M, I)$ and $(A, B) \in$

$\mathscr{B}(G, M, I) \backslash\left\{M_{\emptyset}=\min \{C \mid C \in \mathscr{B}(G, M, I)\}, G_{\emptyset}=\right.$ $\max \{C \mid C \in \mathscr{B}(G, M, I)\}\}$. Then there is one and only one $D_{\gamma_{0}}$ satisfying $A \cap V\left(D_{\gamma_{0}}\right) \neq \emptyset$. Similarly, there is one and only one $D_{\gamma_{1}}$ satisfying $B \cap V\left(D_{\gamma_{1}}\right) \neq \emptyset$. Further, there is one and only one $D_{\gamma_{01}}$ such that $A, B$ belong to $V\left(D_{\gamma_{01}}\right)$.

Proof The existence of $D_{\gamma_{0}}$ for $A$ is carried out by Definition 2 and Definition 4.

Suppose there are two components $D_{1}$ and $D_{2}$ of $D_{I}$ satisfying $A \cap V\left(D_{t}\right) \neq \emptyset,(t=1,2)$.

Let $a_{t} \in A \cap V\left(D_{t}\right)=A_{t},(t=1,2)$. Since $A^{\prime}=B=$ $\{b \in M \mid \forall x \in A, x I b\}=\left\{b \in M \mid \forall x \in A, x b \in E\left(D_{I}\right)\right\}$ by 
Definition 1 and Definition 4. Especially, for $a_{t} \in A,(t=1,2)$, there is $a_{t} b \in E\left(D_{I}\right)$ for any $b \in B,(t=1,2)$. However, by the given, $B \neq \emptyset$. We could put $b_{0} \in B$. There are $a_{1} b_{0}, a_{2} b_{0} \in E\left(D_{I}\right)$. Further, $a_{1}\left(a_{1} b_{0}\right) b_{0}\left(b_{0} a_{2}\right) a_{2}$ is an $\left(a_{1}, a_{2}\right)$-path.

Since $D_{t}=D\left[V\left(D_{t}\right)\right]$ is connected, i.e, for all $x_{t} \in V\left(D_{t}\right) \backslash a_{t}$, there is an $\left(x_{t}, a_{t}\right)$-path to connect $x_{t}$ and $a_{t}, \quad(t=1,2)$. Thus, $\left(x_{1}, a_{1}\right)$-path, $\left(a_{1}, a_{2}\right)$-path, $\left(a_{2}, x_{2}\right)$-path taken together is an $\left(x_{1}, x_{2}\right)$-path to connect $x_{1}$ and $x_{2}$.

By the above, we have the connectivity of $D_{1} \cup D_{2}$, a contradiction to the assumption.

Similarly, there is one and only one component $D_{\gamma_{1}}$ satisfying $B \cap V\left(D_{\gamma_{1}}\right) \neq \emptyset$.

By the construction of $D_{I},(A, B) \in \mathscr{B}(G, M, I)$ and Definition 1 , we have, there is one and only one $D_{\gamma_{01}}$ satisfying $A, B \in V\left(D_{\gamma_{01}}\right)$.

Suppose $(A, B),(X, Y) \in \mathscr{B}(G, M, I) \backslash\left\{M_{\emptyset}, G_{\emptyset}\right\}$ satisfy $(A, B)<(X, Y)$. Then by Definition $1, A \subset X$ and $Y \subset B . Y \subset B$ tells us $Y \subseteq V\left(D_{B}\right)$, where $B \subseteq V\left(D_{B}\right)$ and $D_{B}$ is a component of $D_{I}$. In virtue of Lemma 2, one gets $X \subseteq V\left(D_{B}\right)$ and $A \subseteq V\left(D_{B}\right)$. That is to say, if $(A, B),(X, Y) \in \mathscr{B}(G, M, I)$ and $(A, B),(X, Y)$ are comparable in $\mathscr{B}(G, M, I)$, it must have that $A, B, X$ and $Y$ belong to the same component in $D_{I}$.

About $D_{I}$, we have the following extreme statuses to explain.

Status 1. $y \in M$ and $N(y)=\emptyset$.

By Definition 1 , for $(A, B) \in \mathscr{B}(G, M, I)$ and $B \neq M$, it has $y \notin B$. Namely, for $(A, B) \in \mathscr{B}(G, M, I), y \in B$ if and only if $B=M$.

Status 2. $x \in G$ and $N(x)=\emptyset$.

In virtue of Definition 1 , for $(A, B) \in \mathscr{B}(G, M, I)$ and $A \neq G$, it has $x \notin A$. Namely, for $(A, B) \in \mathscr{B}(G, M, I)$, $x \in A$ if and only if $A=G$.

Status 3. $\left\{g_{m_{1}}, g_{m_{2}}, \ldots, g_{m_{s}}\right\} \subseteq G \quad$ satisfying $N\left(g_{m_{1}}\right)=N\left(g_{m_{2}}\right)=\ldots=N\left(g_{m_{s}}\right)=M$.

In light of Definition 1 , for $(A, B) \in \mathscr{B}(G, M, I)$, it has $g_{m_{i}} \in A,(i=1,2, \ldots, s)$. That is,

$\left(\left\{g_{m_{1}}, g_{m_{2}}, \ldots, g_{m_{s}}\right\}, M\right) \in \mathscr{B}(G, M, I)$.

Status 4. $\left\{m_{n_{1}}, m_{n_{2}}, \ldots, m_{n_{t}}\right\} \subseteq M \quad$ satisfying $N\left(m_{n_{1}}\right)=N\left(m_{n_{2}}\right)=\ldots=N\left(m_{n_{t}}\right)=G$.

Owing to Definition 1 , for $(A, B) \in \mathscr{B}(G, M, I)$, it has $m_{n_{i}} \in A,(i=1,2, \ldots, t)$.

Say, $\left(G,\left\{m_{n_{1}}, m_{n_{2}}, \ldots, m_{n_{t}}\right\}\right) \in \mathscr{B}(G, M, I)$.

According to the Statuses, we assure:

(1) Under the supposition of Status 3, $(A, B) \in \mathscr{B}(G, M, I) \quad$ if and only if $\left(A \backslash\left\{g_{m_{1}}, g_{m_{2}}, \ldots, g_{m_{s}}\right\}, B\right)$ $\mathscr{B}\left(G \backslash\left\{g_{m_{1}}, g_{m_{2}}, \ldots, g_{m_{s}}\right\}, M, I_{1}\right)$,

where $(X, Y) \in I_{1} \Leftrightarrow\left(X \cup\left\{g_{m_{1}}, g_{m_{2}}, \ldots, g_{m_{s}}\right\}, Y\right) \in I$.

(2) Under the supposition of Status $(A, B) \in \mathscr{B}(G, M, I) \quad$ if and only $\left(A, B \backslash \quad\left\{m_{n_{1}}, m_{n_{2}}, \ldots, m_{n_{t}}\right\}\right)$ $\mathscr{B}\left(G, M \backslash\left\{m_{n_{1}}, m_{n_{2}}, \ldots, m_{n_{t}}\right\}, I_{2}\right)$, where $(X, Y) \in I_{2} \Leftrightarrow\left(X, Y \cup\left\{m_{n_{1}}, m_{n_{2}}, \ldots, m_{n_{t}}\right\}\right) \in I$.

Therefore, in Section 3 and the part of 5.1 in Section 5, we only consider $(G, M, I)$ with the property: for $\forall g \in$ $G$, there is $m \in M$ satisfying $(g, m) \notin I$, and at the same time, for $\forall m_{0} \in M$, there is $g_{0} \in G$ satisfying $\left(g_{0}, m_{0}\right) \notin I$. Under such supposition, in $\mathscr{B}(G, M, I),(\emptyset, M)$ and $(G, \emptyset)$ are known existed as the minimum $M_{\emptyset}$ and the maximum $G_{\emptyset}$ respectively.

In $\mathscr{B}(G, M, I)$, we call $M_{\emptyset}$ and $G_{\emptyset}$ trivial elements the others nontrivial elements.

\section{The cover elements of the minimum in $\mathscr{B}(G, M, I)$}

The main aim of studying on $\mathscr{B}(G, M, I)$ is to search nontrivial elements and the relationships among the members in $\mathscr{B}(G, M, I)$.

In this section, we only consider that $D_{I}$ is connected. We will present a way to find all the cover elements of $(\emptyset, M)$ in the concept lattice $\mathscr{B}(G, M, I)$.

Let $G=\left\{g_{1}, g_{2}, \ldots, g_{k}\right\}$. In $D_{I}$, the degree sequence $\left(d\left(g_{1}\right), d\left(g_{2}\right), \ldots, d\left(g_{k}\right)\right)$ satisfies $d\left(g_{1_{1}}\right)=d\left(g_{1_{2}}\right)=\ldots=$ $d\left(g_{1_{t_{1}}}\right)=\min \left\{d\left(g_{1}\right), d\left(g_{2}\right), \ldots, d\left(g_{k}\right)\right\}<d\left(g_{2_{1}}\right)=$ $d\left(g_{2_{2}}\right)=\ldots=d\left(g_{2_{t_{2}}}\right)<\ldots<d\left(g_{i_{1}}\right)=d\left(g_{i_{2}}\right)=\ldots=$ $d\left(g_{i_{t_{i}}}\right)<d\left(g_{(i+1)_{1}}\right) \stackrel{=}{=} d\left(g_{(i+1)_{2}}\right)=\ldots=d\left(g_{(i+1)_{t_{i+1}}}\right)<$ $\ldots<d\left(g_{s_{1}}\right)=d\left(g_{s_{2}}\right)=\ldots=d\left(g_{s_{s}}\right)=$ $\max \left\{d\left(g_{1}\right), d\left(g_{2}\right), \ldots, d\left(g_{k}\right)\right\}$,

where for any $g_{j} \in G, d\left(g_{i_{1}}\right)<d\left(g_{j}\right)<d\left(g_{(i+1)_{1}}\right)$ is not true.

This sequence and $D_{I}$ have some remarkable properties shown as follows.

Lemma 3 (1) $0 \leq d\left(g_{j}\right),(j=1,2, \ldots, k)$.

(2) $d\left(g_{j}\right)=\left|N\left(g_{j}\right)\right|,(j=1,2, \ldots, k)$.

(3) For any $g_{j} \in G$, if $G \ni g \in N\left(g_{j}\right)^{\prime}$, then $N\left(g_{j}\right) \subseteq$ $N(g),(j=1,2, \ldots, k)$.

(4) $(X, Y) \in \mathscr{B}(G, M, I)$ induces $Y=\bigcap_{x \in X} N(x)$.

Proof (1) and (2) are got by Definition 2 and Definition 4.

Let $g \in N\left(g_{j}\right)^{\prime}$. Since $N\left(g_{j}\right)^{\prime}=\{x \in G$ । $\left.\forall y \in N\left(g_{j}\right), x I y\right\}$ by Definition 1 , we have $g I y$ for $y \in N\left(g_{j}\right)$, and in view of Definition 4, gy $\in E\left(D_{I}\right)$ for every $y \in N\left(g_{j}\right)$. Hence, owing to Definition 2, $N\left(g_{j}\right) \subseteq N(g)$. Say, (3) is true.

By Definition 1 and Definition 2, it is easy to have $Y \subseteq$ $N(x)$, and so $Y \subseteq \bigcap_{x \in X} N(x)$.

If $Y \subset \bigcap_{x \in X} N(x)$, that is, there is $b \in \bigcap_{x \in X} N(x) \backslash Y$. This implies $b \in N(x)$ for $x \in X$, say, $x b \in E\left(D_{I}\right)$ for every $x \in X$. Thus, $b \in X^{\prime}$. However, $X^{\prime}=Y$ holds according to $(X, Y) \in \mathscr{B}(G, M, I)$ and Definition 1. This follows $b \in Y$, a contradiction with $b \notin Y$. Hence, $Y=\bigcap_{x \in X} N(x)$, i.e. (4) 
is correct.

We are now ready to prove our main results.

Theorem 1 Let $g_{j} \in G$. Then $\left(N\left(g_{j}\right)^{\prime}, N\left(g_{j}\right)\right) \in \mathscr{B}(G, M, I),(j=1,2, \ldots, k)$.

Proof By Lemma $1, N\left(g_{j}\right) \subseteq N\left(g_{j}\right)^{\prime \prime}$ holds. According to Definition 1, it only needs to prove $N\left(g_{j}\right)^{\prime \prime} \subseteq N\left(g_{j}\right)$.

In light of Definition 1 and Definition 4 , one gets $N\left(g_{j}\right)^{\prime \prime}=\left\{y \in M \mid \forall x \in N\left(g_{j}\right)^{\prime}, x y \in E\left(D_{I}\right)\right\}$ and $N\left(g_{j}\right)^{\prime}=\left\{a \in G \mid \forall b \in N\left(g_{j}\right), a b \in E\left(D_{I}\right)\right\}$.

By Definition 2 and Definition $4, g_{j} b \in E\left(D_{I}\right)$ holds for $\forall b \in N\left(g_{j}\right)$. It follows $g_{j} \in N\left(g_{j}\right)^{\prime}$. Hence, $g_{j} y \in E\left(D_{I}\right)$ for $\forall y \in N\left(g_{j}\right)^{\prime \prime}$. So $y \in N\left(g_{j}\right)$. Namely, $N\left(g_{j}\right)^{\prime \prime} \subseteq N\left(g_{j}\right)$.

That is to say, $\left(N\left(g_{j}\right)^{\prime}, N\left(g_{j}\right)\right) \in \mathscr{B}(G, M, I)$.

Theorem 2 Let $g \in G, p \in\left\{1, \ldots, t_{s}\right\}$ and $d\left(g_{s_{p}}\right)=$ $\max \left\{d\left(g_{1}\right), \ldots, d\left(g_{k}\right)\right\}$. Then

(1) $N(g) \cap N\left(g_{s_{p}}\right)=N\left(g_{s_{p}}\right) \Longleftrightarrow g \in N\left(g_{s_{p}}\right)^{\prime}$.

(2) $\left(N\left(g_{s_{p}}\right)^{\prime}, N\left(g_{s_{p}}\right)\right)$ covers $(\emptyset, M)$ in $\mathscr{B}(G, M, I)$.

Proof $(1)(\Rightarrow) N(g) \cap N\left(g_{s_{p}}\right)=N\left(g_{s_{p}}\right)$ hints

$N\left(g_{s_{p}}\right) \subseteq N(g)$. In light of Lemma 3, this implies $d\left(g_{s_{p}}\right)=$ $\left|N\left(g_{s_{p}}\right)\right| \leq|N(g)|=d(g)$. However, $d\left(g_{s_{p}}\right)=$

$\max \left\{d\left(g_{1}\right), d\left(g_{2}\right), \ldots, d\left(g_{k}\right)\right\}$. Thus $d(g)=d\left(g_{s_{p}}\right)$ is true, further, $N\left(g_{s_{p}}\right)=N(g)$ holds. Herein, $g \in N(g)^{\prime}=N\left(g_{s_{p}}\right)^{\prime}$.

$(\Leftarrow) g \in N\left(g_{s_{p}}\right)^{\prime}$ and Lemma 3 together hints $N\left(g_{s_{p}}\right) \subseteq$ $N(g)$, i.e $N\left(g_{s_{p}}\right)=N\left(g_{s_{p}}\right) \cap N(g)$.

(2) In view of Theorem $1,\left(N\left(g_{s_{p}}\right)^{\prime}, N\left(g_{s_{p}}\right)\right) \in$ $\mathscr{B}(G, M, I)$.

Suppose there is $(X, Y) \in \mathscr{B}(G, M, I)$ satisfying $(\emptyset, M)<(X, Y)<\left(N\left(g_{s_{p}}\right)^{\prime}, N\left(g_{s_{p}}\right)\right)$. It has $\emptyset \neq X \subset N\left(g_{s_{p}}\right)^{\prime}$ and $N\left(g_{s_{p}}\right) \subset Y \subset M$. Let $y_{0} \in Y \backslash N\left(g_{s_{p}}\right) \neq \emptyset$.

Because $X=Y^{\prime}=\{x \in G \mid \forall y \in Y, x I y\}=\{x \in G \mid \forall y \in$ $\left.Y, x y \in E\left(D_{I}\right)\right\}$. Then, for $y_{0} \in Y$ and $\forall x \in Y^{\prime}=X$, there is $x y_{0} \in E\left(D_{I}\right)$. This follows $y_{0} \in N(x)$ for $\forall x \in X$. However, $x \in X \subset N\left(g_{s_{p}}\right)^{\prime}$ and the above closed (1) together shows us $N(x)=N\left(g_{s_{p}}\right)$. Thus $y_{0} \in N\left(g_{s_{p}}\right)$, a contradiction to $y_{0} \in Y \backslash N\left(g_{s_{p}}\right)$.

That is to say, $\left(N\left(g_{s_{p}}\right)^{\prime}, N\left(g_{s_{p}}\right)\right)$ covers $(\emptyset, M)$.

Theorem 2 endows the cover elements of $(\emptyset, M)$ yielded from the members in $\mathscr{T}_{s}=\left\{g_{s_{1}}, g_{s_{2}}, \ldots, g_{s_{t_{s}}}\right\}$. We will now discuss how to find the other cover elements of $(\emptyset, M)$.

Suppose we have got the cover elements of $(\emptyset, M)$ yielded from

$\mathscr{T}_{i+1}=\left\{g_{(i+1)_{1}}, g_{(i+1)_{2}}, \ldots, g_{(i+1)_{i+1}}\right\} \backslash\left\{g_{j} \in G \mid d\left(g_{j}\right)=\right.$ $d\left(g_{(i+1)_{1}}\right)$, additionally, there is $g \in G$ satisfying $\left.N\left(g_{j}\right) \subset N(g)\right\}$. Put

$\mathscr{T}_{i}=\left\{g_{i_{1}}, g_{i_{2}}, \ldots, g_{i_{t_{i}}}\right\} \backslash\left\{g_{j} \in G \mid d\left(g_{j}\right)=d\left(g_{i_{1}}\right)\right.$, there is

$g \in G$ satisfying $\left.N\left(g_{j}\right) \subset N(g)\right\}$ $=\left\{g_{i_{\alpha_{1}}}, g_{i_{\alpha_{2}}}, \ldots, g_{i_{\alpha_{i}}}\right\}$.

Then we get a sequence $\mathscr{T}_{1}, \mathscr{T}_{2}, \ldots, \mathscr{T}_{s}$. Considering with Theorem 1, we will prove that the following Theorem 3 is true.

Theorem $3 \quad\left(N\left(g_{i_{\alpha_{h}}}\right)^{\prime}, N\left(g_{i_{\alpha_{h}}}\right)\right)$ covers $(\emptyset, M)$, $\left(h=1,2, \ldots, \beta_{i}\right)$.

Proof Otherwise, there is $(X, Y) \in \mathscr{B}(G, M, I)$ satisfying $(\emptyset, M)<(X, Y)<\left(N\left(g_{i_{\alpha_{h}}}\right)^{\prime}, N\left(g_{i_{\alpha_{h}}}\right)\right)$.

In view of Definition $1, N\left(g_{i_{\alpha_{h}}}\right) \subset Y$ and $\emptyset \neq X \subset N\left(g_{i_{\alpha_{h}}}\right)^{\prime}$ are correct.

Put $a \in N\left(g_{i_{\alpha_{h}}}\right)^{\prime}$. It has $\{a\}^{\prime} \supseteq N\left(g_{i_{\alpha_{h}}}\right)^{\prime \prime}=N\left(g_{i_{\alpha_{h}}}\right)$. According to Definition 1 and Section 2, $\{a\}^{\prime}=\{y \in M \mid \forall x \in\{a\}, x I y\}=\{y \in M \mid a I y\}=\{y \in$ $M \mid$ ay $\left.\in E\left(D_{I}\right)\right\}=N(a)$. Thus, $N\left(g_{i_{\alpha_{h}}}\right) \subseteq N(a)$.

If $N\left(g_{i_{\alpha_{h}}}\right) \subset N(a)$ for some $a_{0} \in N\left(g_{i_{\alpha_{h}}}\right)^{\prime}$. This causes a contradiction to the choice of $g_{i_{\alpha_{h}}}$. That is to say, $N(a)=$ $N\left(g_{i_{\alpha_{h}}}\right)$ for any $a \in N\left(g_{i_{\alpha_{h}}}\right)^{\prime}$. Particularly, $x \in X \subset N\left(g_{i_{\alpha_{h}}}\right)^{\prime}$ leads to $N(x)=N\left(g_{i_{\alpha_{h}}}\right)$.

Thus by $(X, Y) \in \mathscr{B}(G, M, I)$ and Lemma 3 , it follows $Y=\bigcap_{x \in X} N(x)=\bigcap_{x \in X} N\left(g_{i_{\alpha_{h}}}\right)=N\left(g_{i_{\alpha_{h}}}\right)$, a contradiction to $N\left(g_{i_{h}}\right) \subset Y$.

Therefore, $\left(N\left(g_{i_{\alpha_{h}}}\right)^{\prime}, N\left(g_{i_{\alpha_{h}}}\right)\right) \operatorname{covers}(\emptyset, M),\left(h=1,2, \ldots, \beta_{i}\right)$. Observing $\mathscr{T}_{1}, \ldots, \mathscr{T}_{s}$, Theorem 2 and Theorem 3 , by the induction on $s \leq|G|<\infty$, we obtain that the style members $\left(N(g)^{\prime}, N(g)\right)$ covers $(\emptyset, M)$, where $g \in G$, additionally, for any $x \in G, N(g) \subset N(x)$ is wrong.

We will now discuss the converse part of the above closed result as follows.

Theorem 4 Let $(X, Y)$ cover $(\emptyset, M)$ in $\mathscr{B}(G, M, I)$. Then there is $g_{Y} \in G$ such that $(X, Y)=\left(N\left(g_{Y}\right)^{\prime}, N\left(g_{Y}\right)\right)$ and for any $g \in G, N\left(g_{Y}\right) \subset N(g)$ is wrong.

Proof That $(X, Y)$ covers $(\emptyset, M)$ hints $\emptyset \neq X \subseteq G$ and $Y \subset M$.

Let $a \in X$. Because $X^{\prime}=Y=\{y \in M \mid \forall x \in X, x I y\}=$ $\left\{y \in M \mid \forall x \in X, x y \in E\left(D_{I}\right)\right\}$, in particular, $a y \in E\left(D_{I}\right)$ for any $y \in Y$. This implies $y \in N(a)$ for $\forall y \in Y$, i.e. $Y \subseteq N(a)$.

Suppose $Y \subset N(a)$ for any $a \in X$.

Since $\left(N(a)^{\prime}, N(a)\right) \in \mathscr{B}(G, M, I)$ is correct by Theorem 1. Further, $\left(N(a)^{\prime}, N(a)\right)<(X, Y)$ holds by the supposition and Definition 1. These and $(X, Y)$ covers $(\emptyset, M)$ taken together will bring about $\left(N(a)^{\prime}, N(a)\right)=(\emptyset, M)$. That is $N(a)=M$.

But $Y=\bigcap_{x \in X} N(x)$ holds according to Lemma 3 . Considering the arbitrary of $a \in X$ and the above discussion, we have $Y=M$, a contradiction to $Y \subset M$.

That is to say, $Y=N\left(g_{Y}\right)$ for some $g_{Y} \in G$. Herein, $(X, Y)=\left(N\left(g_{Y}\right)^{\prime}, N\left(g_{Y}\right)\right)$.

On the other hand, suppose there is $g_{0} \in G$ satisfying $N\left(g_{Y}\right) \quad \subset \quad N\left(g_{0}\right) . \quad$ By $\quad$ Theorem 1, $\left(N\left(g_{0}\right)^{\prime}, N\left(g_{0}\right)\right) \in \mathscr{B}(G, M, I)$. In view of Definition 1, it has $(X, Y)=\left(N\left(g_{Y}\right)^{\prime}, N\left(g_{Y}\right)\right)>\left(N\left(g_{0}\right)^{\prime}, N\left(g_{0}\right)\right)$. 
However, that $(X, Y)$ covers $(\emptyset, M)$ compels $(\emptyset, M)=\left(N\left(g_{0}\right)^{\prime}, N\left(g_{0}\right)\right)$, and so $\emptyset=N\left(g_{0}\right)^{\prime}$.

Here, we should notice that $g_{0} \in N\left(g_{0}\right)^{\prime}$ is right owing to Definition 1 . Namely, $N\left(g_{0}\right)^{\prime} \neq \emptyset$ is correct. This follows a contradiction to $\emptyset=N\left(g_{0}\right)^{\prime}$.

In one word, there does not exist any $g \in G$ satisfying $N\left(g_{Y}\right) \subset N(g)$.

The following approach is the sketch of an algorithm to obtain the cover elements of $(\emptyset, M)$ yielded from $\mathscr{T}_{s}=$ $\left\{g_{s_{1}}, g_{s_{2}}, \ldots, g_{s_{t_{s}}}\right\}$.

Step 1. Let $\mathscr{H}=\emptyset, \mathscr{C}_{s}=\emptyset$ and $\mathscr{T}=\left\{1,2, \ldots, t_{s}\right\}$.

Step 2. If $\mathscr{T} \neq \emptyset$, then $\mathscr{H}=\mathscr{T}$, go to Step 3 .

Otherwise, go to Step 7.

Step 3. $\xi=\min \mathscr{H}, N\left(g_{s_{\xi}}\right)^{\prime}=\emptyset$ and $N_{s_{\xi}}^{\prime}=\emptyset$.

Step 4. If $\mathscr{H} \neq \emptyset$, then $\alpha=\min \mathscr{H}$, go to Step 5 .

Otherwise, go to step 6.

Step 5. If $N\left(g_{s_{\xi}}\right) \cap N\left(g_{s_{\alpha}}\right)=N\left(g_{s_{\xi}}\right)$, then

$N_{s_{\xi}}^{\prime}=N_{s_{\xi}}^{\prime} \cup g_{s_{\alpha}}$ and $\mathscr{H}=\mathscr{H} \backslash \alpha$, go to Step 4.

Otherwise, $\mathscr{H}=\mathscr{H} \backslash \alpha$ and $\mathscr{T}=(\mathscr{T} \backslash \xi) \cup \alpha$, go to Step 4.

Step 6. $N\left(g_{s_{\xi}}\right)^{\prime}=N_{s_{\xi}}^{\prime}, \mathscr{C}_{s}=\mathscr{C}_{s} \cup\left\{\left(N\left(g_{s_{\xi}}\right)^{\prime}, N\left(g_{s_{\xi}}\right)\right)\right\}$, go to Step 2.

Step 7. Stop.

By the above algorithm, we get that $\mathscr{C}_{s}$ is the need cover elements.

Suppose we have got the set $\mathscr{C}_{i+1}$ of the cover elements of $(\emptyset, M)$ yielded from

$\mathscr{T}_{i+1}=\left\{g_{(i+1)_{1}}, g_{(i+1)_{2}}, \ldots, g_{(i+1)_{t_{i+1}}}\right\} \backslash\left\{g_{j} \in G \mid d\left(g_{j}\right)=\right.$ $d\left(g_{\left.(i+1)_{1}\right)}\right)$ besides, there is $g \in G$ satisfying $\left.N\left(g_{j}\right) \subset N(g)\right\}$.

Put $\mathscr{T}_{i}=\left\{g_{i_{1}}, g_{i_{2}}, \ldots, g_{i_{i}}\right\} \backslash\left\{g_{j} \in G \mid d\left(g_{j}\right)=d\left(g_{i_{1}}\right)\right.$, besides, there is $g \in G$ satisfying $\left.N\left(g_{j}\right) \subset N(g)\right\}=\left\{g_{i_{\alpha_{1}}}, g_{i_{\alpha_{2}}}, \ldots, g_{i_{\alpha_{\beta_{i}}}}\right\}$.

If $\mathscr{T}_{i} \neq \emptyset$, then posit $\mathscr{T}=\left\{\alpha_{1}, \alpha_{2}, \ldots, \alpha_{\beta_{i}}\right\}$ and repeat the algorithm above for $\mathscr{T}$, we obtain $\mathscr{C}_{i}$, the set of the cover elements of $(\emptyset, M)$ yielded from $\mathscr{T}_{i}$.

If $\mathscr{T}_{i}=\emptyset$, then $\mathscr{C}_{i}=\emptyset$. We just consider $\mathscr{T}_{\eta}$ successively, $\quad \eta=i-1, i-2, \ldots, 1$, where $i-1>i-2>\ldots>1$.

According to $\left|\mathscr{T}_{i}\right| \leq|G|<\infty,(i=1,2, \ldots, s \leq|G|)$ and the Theorems from Theorem 1 to Theorem 4 , it brings about that the set $\mathscr{C}$ of the cover elements of $(\emptyset, M)$ is $\mathscr{C}=\mathscr{C}_{1} \cup \mathscr{C}_{2} \cup \ldots \cup \mathscr{C}_{s}$.

\section{The covers of an element of the lattice}

We will use the classical properties of lattice theory and graph theory to construct a one-to-one correspondence between an interval of the lattice and the minimum element in the concept lattice of a new binary relation.
At first, we examine what happens to $(A, B) \in \mathscr{B}(G, M, I)$ when a new underlying graph is established. In Definition 5, we will not suppose that $D_{I}$ is always connected and Status 3 or Status 4 in Section 2 could not happen.

Definition 5 Let $(A, B) \in \mathscr{B}(G, M, I)$; we will define a new binary relation $I_{A B} \subseteq(G \backslash A) \times B$, as follows:

$\left(X_{A}, Y_{A}\right) \in I_{A B} \Leftrightarrow\left(X_{A} \cup A, Y_{A}\right)=(X, Y) \in I$ where $X_{A}=X \backslash A$ and $Y_{A}=Y$.

For the sake of convenience, before Theorem 5, we just suppose that none of Status 3 and Status 4 in Section 2 will happen here.

Note that $(X, Y) \in[(A, B),(G, \emptyset)] \subseteq \mathscr{B}(G, M, I)$ tells us $A \subseteq X \subseteq G, Y \subseteq B$ and $(X, Y) \in I$. Simultaneously, it also tells us $X \backslash A \subseteq G \backslash A, Y \subseteq B$ and $(X \backslash A, Y) \in I_{A B}$. By Definition 5, it follows that $\left(X_{A}, Y_{A}\right) \in I_{A B}$ induces $(X, Y)=\left(X_{A} \cup A, Y\right) \in[(A, B),(G, \emptyset)]$.

Analogously to the construction of $D_{I}$ in Section 2, the associated underlying graph $D_{I_{A B}}$ with $\left(G \backslash A, B, I_{A B}\right)$ is provided.

We firstly remark that by virtue of Definition $1,(G \backslash$ $\left.A, B, I_{A B}\right)$ is a new context and $(\emptyset, B)$ is the minimum in $\mathscr{B}\left(G \backslash A, B, I_{A B}\right)$.

Posit $\left(X_{1}, Y_{1}\right)$ be a cover element of $(\emptyset, B)$ in $\mathscr{B}(G \backslash$ $\left.A, B, I_{A B}\right)$. Then one gets $\emptyset \neq X_{1}, Y_{1} \subset B$ and $\left(X_{1}, Y_{1}\right) \in I_{A B}$, and further, $\left(X_{2}, Y_{2}\right) \in[(A, B),(G, \emptyset)] \subseteq \mathscr{B}(G, M, I)$ where $X_{2}=X_{1} \cup A$ and $Y_{2}=Y_{1}$.

Suppose $\left(X_{2}, Y_{2}\right)$ does not cover $(A, B)$ in $\mathscr{B}(G, M, I)$. We will get that there is $\left(X_{3}, Y_{3}\right) \in \mathscr{B}(G, M, I)$ satisfying $(A, B)<\left(X_{3}, Y_{3}\right)<\left(X_{2}, Y_{2}\right)$. In view of Definition 5 and the above discussion, $(\emptyset, B)<\left(X_{3} \backslash A, Y_{3}\right)<\left(X_{2} \backslash A, Y_{2}\right)=$ $\left(X_{1}, Y_{1}\right)$ is correct in $\mathscr{B}\left(G \backslash A, B, I_{A B}\right)$. This brings about a contradiction to the position of $\left(X_{1}, Y_{1}\right)$ in $\mathscr{B}\left(G \backslash A, B, I_{A B}\right)$. Thus, $\left(X_{2}, Y_{2}\right)$ covers $(A, B)$ in $\mathscr{B}(G, M, I)$.

Likewise, if $(X, Y)$ covers $(A, B)$ in $\mathscr{B}(G, M, I)$, then it must have $(X \backslash A, Y)$ covers $(\emptyset, B)$ in $\mathscr{B}\left(G \backslash A, B, I_{A B}\right)$.

Summary, one will get a result as follows: $(X, Y) \in \mathscr{B}(G, M, I)$ covers $(A, B)$ in $\mathscr{B}(G, M, I)$ if and only if $(X \backslash A, Y) \in \mathscr{B}\left(G \backslash A, B, I_{A B}\right)$ covers $(\emptyset, B)$ in $\mathscr{B}\left(G \backslash A, B, I_{A B}\right)$.

The below in this Section, we will not suppose that both Status 3 and Status 4 will not happen; that $D_{I}$ is connected will not assumed. Considering the above closed result and the study on the Statuses in Section 2, we pledge that the following Theorem 5 is valid.

Theorem $5(X, Y) \in \mathscr{B}(G, M, I)$ covers $(A, B)$ in $\mathscr{B}(G, M, I)$ if and only if $(X \backslash A, Y) \in \mathscr{B}\left(G \backslash A, B, I_{A B}\right)$ covers $\quad B_{\emptyset}$ in $\mathscr{B}\left(G \backslash A, B, I_{A B}\right)$, where $B_{\emptyset}$ is $\min \left\{C \mid C \in \mathscr{B}\left(G \backslash A, B, I_{A B}\right)\right\}$. 


\section{Generating the lattice}

Computing the cover elements of an element in $\mathscr{B}(G, M, I)$ is an important problem for finding out all the concepts of a given context and the diagram of $\mathscr{B}(G, M, I)$. One may generate all the concepts defined by a binary relation, and at the same time, its diagram.

\subsection{The first process}

In the lattice $\mathscr{B}(G, M, I)$, if the height function is $h$, by [3], we will know $h(a)=h(b)+1$ when $a \in \mathscr{B}(G, M, I)$ covers $b \in \mathscr{B}(G, M, I)$. Because the relations we work on are considered as non-empty, one gets $h(G, \emptyset) \geq 1$.

When $h(G, \emptyset)=1 . \mathscr{B}(G, M, I)$ has only the two trivial elements.

When $h(G, \emptyset)>1$. We have $0=h(\emptyset, M)<h(X, Y)<$ $h(G, \emptyset)<\infty$ for any nontrivial element $(X, Y) \in \mathscr{B}(G, M, I)$.

Applying the method in Section 3, we will have all the cover elements $\mathbf{F}_{(\emptyset, M)}$ of $(\emptyset, M)$. In addition, $h(X, Y)=1 \Leftrightarrow(X, Y)$ covers $(\emptyset, M)$.

Suppose there are at least two components $D\left[V_{1}\right]$ and $D\left[V_{2}\right]$ of $D_{I}$. Let $\left|V_{2}\right|>1$ and $V_{1} \cap G=V_{1}$ with $\left|V_{1}\right|=1$. $1<\left|V_{2}\right|$ and $D\left[V_{2}\right]$ is connected taken together hints $\left|E\left(V_{2}\right)\right| \geq 1$. We will prove the following Lemma 4 .

Lemma 4 Any concept yielded from $D\left[V_{1}\right]$ will not cover $(\emptyset, M)$ if there is another component $D\left[V_{2}\right]$ satisfying $\left|V_{2}\right|>1$.

Proof For any $(A, B) \in \mathscr{B}(G, M, I)$, Lemma 2 says that $A, B$ belong to the same component. Let $V_{1}=\left\{v_{1}\right\}$ and $V_{2}=\left\{v_{2}, v_{3}, \ldots, v_{\eta_{2}}\right\} \cup\left\{w_{2}, w_{3}, \ldots, w_{\varphi_{2}}\right\}$, where $\left\{v_{2}, v_{3}, \ldots, v_{\eta_{2}}\right\} \subseteq G$ and $\left\{w_{2}, w_{3}, \ldots, w_{\varphi_{2}}\right\} \subseteq M$.

It is easy to see that in $D_{I}, N\left(v_{1}\right)=\emptyset$ is valid. Recalled on Status 2 in Section 2, this leads that there is only one concept $(G, \emptyset)$ yielded from $D\left[V_{1}\right]$.

On the other hand, by the results in Section 3, $\left(N\left(v_{\pi}\right)^{\prime}, N\left(v_{\pi}\right)\right) \in \mathscr{B}(G, M, I)$ covers $(\emptyset, M)$, where $d\left(v_{\pi}\right)=\max \left\{d\left(v_{2}\right), d\left(v_{3}\right), \ldots, d\left(v_{\eta_{2}}\right)\right\}$.

By virtue of $\left|E\left(V_{2}\right)\right| \geq 1$, one gets that $d\left(v_{\pi}\right) \geq 1$ is effective, and so $\left|N\left(v_{\pi}\right)\right| \geq 1$. Herein, $N\left(v_{\pi}\right) \neq \emptyset$ is right. Thus, $\left(N\left(v_{\pi}\right)^{\prime}, N\left(v_{\pi}\right)\right)<(G, \emptyset)=\left(N\left(v_{1}\right)^{\prime}, N\left(v_{1}\right)\right)$.

By the knowledge of lattice theory, here, $(G, \emptyset)$ will not cover $(\emptyset, M)$.

Based on Lemma 4, we get that if all the components $D_{1}, D_{2}, \ldots, D_{\gamma} \quad$ of $\quad D_{I} \quad$ satisfy $\quad\left|V\left(D_{1}\right) \cap G\right|=$ $\left|V\left(D_{2}\right) \cap G\right|=\ldots=\left|V\left(D_{\gamma}\right) \cap G\right|=1$, then there is only $(G, \emptyset)$ belonging to the set of cover elements of $(\emptyset, M)$. In another word to say, for $(A, B) \in \mathscr{B}(G, M, I)$ and $A \neq \emptyset$, the underlying graph $D_{I_{A B}}$ associated to $(A, B)$ satisfies

$E\left(D_{I_{A B}}\right)=\emptyset$ if and only if $(A, B)$ is $(G, \emptyset)$.

Considering with Lemma 4 , for each component $D_{t}$ of $D_{I}$, we can use the results for Statuses in Section 2 and the manner in Section 3 to obtain the covers $\mathscr{C}_{t}$ of $(\emptyset, M)$, $(t=1,2, \ldots, \gamma)$. Calling the results in Section 2 back, we have $\mathbf{F}_{(\emptyset, M)}=\bigcup_{t=1}^{\gamma} \mathscr{C}_{t}$

\subsection{The second process}

Beyond now in this Section, we just consider $(G, M, I)$ with the property that for any $g \in G$, there is $m \in M$ satisfying $(g, m) \notin I$, and at the same time, for any $m_{0} \in M$, there is $g_{0} \in G$ satisfying $\left(g_{0}, m_{0}\right) \notin I$. But, below, no such supposition exists.

Using the discussions in Section 2 and 5.1, we have

$(X, Y) \in \mathbf{F}_{(\emptyset, M)}^{0} \Leftrightarrow\left(X \cup\left\{g_{m_{1}}, g_{m_{2}}, \ldots, g_{m_{s}}\right\}\right.$,

$\left.Y \cup\left\{m_{n_{1}}, m_{n_{2}}, \ldots, m_{n_{t}}\right\}\right) \in \mathbf{F}_{M_{\emptyset}}$, where $\mathbf{F}_{(\emptyset, M)}^{0}$ is the set of covers of $(\emptyset, M)$ in $\mathscr{B}\left(G \backslash\left\{g_{m_{1}}, g_{m_{2}}, \ldots, g_{m_{s}}\right\}, M \backslash\left\{m_{n_{1}}, m_{n_{2}}, \ldots, m_{n_{t}}\right\}, I_{1}\right) ;$ $\mathbf{F}_{M_{\emptyset}}$ is the set of covers of $M_{\emptyset}$ in $\mathscr{B}(G, M, I)$;

$(U, V) \in I_{1} \Leftrightarrow$

$\left(U \cup\left\{g_{m_{1}}, g_{m_{2}}, \ldots, g_{m_{s}}\right\}, Y \cup\left\{m_{n_{1}}, m_{n_{2}}, \ldots, m_{n_{t}}\right\}\right) \in I ;$

$\left\{g_{m_{1}}, g_{m_{2}}, \ldots, g_{m_{s}}\right\} \subseteq G, N_{D_{I}}\left(g_{m_{1}}\right)=N_{D_{I}}\left(g_{m_{2}}\right)=\ldots=$ $N_{D_{I}}\left(g_{m_{s}}\right)=M$

$\left\{m_{n_{1}}, m_{n_{2}}, \ldots, m_{n_{t}}\right\} \subseteq M, N_{D_{I}}\left(m_{n_{1}}\right)=N_{D_{I}}\left(m_{n_{2}}\right)=\ldots=$ $N_{D_{I}}\left(m_{n_{t}}\right)=G$.

As the talk in 5.1, we can get $\mathbf{F}_{(\emptyset, M)}^{0}$. Considering the above talk, we will obtain $\mathbf{F}_{M_{\emptyset}}$.

Though by Section 2 especially Lemma 2, for $(A, B) \in$ $\mathscr{B}(G, M, I) \backslash\left\{M_{\emptyset}, G_{\emptyset}\right\}$, if $\left(X_{t}, Y_{t}\right) \in \mathscr{B}(G, M, I) \backslash\left\{M_{\emptyset}, G_{\emptyset}\right\}$ covers $(A, B)$, it causes that all of $A, B, X_{t}, Y_{t}$ belong to the same component of $D_{I},(t=1,2)$, we could not say that both the pair of $X_{1} \backslash A$ and $Y_{1}$ and the pair of $X_{2} \backslash A$ and $Y_{2}$ belong to the same component of $D_{I_{A B}}$. The reason is that $D_{I_{A B}}$ is perhaps disconnected.

When we examine the cover elements of $(U, W) \in \mathscr{B}(G, M, I)$. If $D_{I_{U W}}$ is disconnected, in virtue of Section 2, we just separately consider the different components of $D_{I_{U W}}$. For each component in $D_{I_{U W}}$, we use the outcomes from Section 2 and that beyond this Section. The main idea is as the following.

Put $\left(X_{0}, Y_{0}\right)$ be a cover element of $M_{\emptyset}$ and $\left\{D_{q} \mid q=1,2, \ldots, n\right\}$ be the set of components of $D_{I_{X_{0} Y_{0}}}$. Now we just want to search out the family $\mathbf{F}_{\left(X_{0}, Y_{0}\right)}$ of all the cover elements of $\left(X_{0}, Y_{0}\right)$ in $\mathscr{B}(G, M, I)$. For $\left(G \backslash X_{0}, Y_{0}, I_{X_{0} Y_{0}}\right)$, we hope to use the methods appeared in Section 3, Section 4 and that at the above. Unfortunately, we could not pledge that the previous conditions are built on. So we need to consider it under the following two cases to search out the set $\mathbf{F}_{\left(X_{0}, Y_{0}\right)}$ of covers of $\left(X_{0}, Y_{0}\right)$ in $\mathscr{B}(G, M, I)$.

Case 1. $\left|V\left(D_{q}\right)\right|=1$ for every $q \in\{1,2, \ldots, n\}$.

By the Statuses in Section 2, we will get all the cover elements of $\left(X_{0}, Y_{0}\right)$ in $\mathscr{B}\left(G \backslash X_{0}, Y_{0}, I_{X_{0} Y_{0}}\right)$. Afterwards, under the instruction of Theorem 5 , we obtain the covers of $\left(X_{0}, Y_{0}\right)$ in $\mathscr{B}(G, M, I)$.

Case 2. If there is $D_{\theta}$ satisfying $\left|V\left(D_{\theta}\right)\right|>1$ for some $\theta \in\{1,2, \ldots, n\}$.

By Lemma 4, we need not to consider the component $D_{\delta}$ where $\left|V\left(D_{\delta}\right)\right|=1, \delta \in\{1,2, \ldots, n\}$. Review Status 1 
in Section 2, if $\left|V\left(D_{\psi}\right)\right|=1$ and $V\left(D_{\psi}\right) \cap M \neq \emptyset,(\psi \in$ $\{1, \ldots, n\})$, then when we search the covers of $\left(X_{0}, Y_{0}\right)$, it needs not to consider $D_{\psi}$. Therefore, we only put our attention to the components as $D_{\theta}$ where $\left|V\left(D_{\theta}\right)\right|>1$.

Firstly, because $D_{\theta}$ is connected, the Status 1 and Status 2 must not happen for $D_{\theta}$. If the Status 3 or Status 4 happens, we just use the discussion for the two statuses in Section 2 and Theorem 5 to get the covers of $\left(X_{0}, Y_{0}\right)$ in $\mathscr{B}(G, M, I)$.

For example, the Status 3 happens, i.e. there are $\left\{x_{1}, x_{2}, \ldots, x_{\zeta}\right\} \in V\left(D_{\theta}\right) \cap G$ satisfying $N_{D_{\theta}}\left(X_{t}\right)=$ $V\left(D_{\theta}\right) \cap M$. Then by the result in Section 2,

$\left(\left\{x_{1}, x_{2}, \ldots, x_{\zeta}\right\}, V\left(D_{\theta}\right) \cap M\right)$ is the minimum in $\mathscr{B}\left(G \backslash X_{0}, Y_{0}, I_{X_{0} Y_{0}}\right)$. Further, in light of Theorem 5,

$\left(X_{0} \cup\left\{x_{1}, x_{2}, \ldots, x_{\zeta}\right\}, V\left(D_{\theta}\right) \cap M\right)$ is the only one cover of $\left(X_{0}, Y_{0}\right)$ in $\mathscr{B}(G, M, I)$.

Secondly, if both Status 3 and Status 4 do not happen for $D_{\theta}$.

According to $\left|V\left(D_{\theta}\right)\right|>1$, Lemma 2 and Section 4, we could use the method in Section 3 to find all the cover elements $\mathscr{C}_{\theta}\left(X_{0}, Y_{0}\right)$ of $\left(\emptyset, Y_{0}\right)$ in $\mathscr{B}\left(G \backslash X_{0}, Y_{0}, I_{X_{0} Y_{0}}\right)$ born in $D_{\theta}$.

Under the guide of Theorem 5, we will get the set $\mathbf{H}_{\theta}\left(X_{0}, Y_{0}\right)$ of the cover elements of $\left(X_{0}, Y_{0}\right)$ in $\mathscr{B}(G, M, I)$ associated with $D_{\theta}$.

Finally, considering Lemma 2 and Section 2, it will have the family $\mathbf{F}_{\left(X_{0}, Y_{0}\right)}$. In virtue of Section 2 , we have $\mathbf{F}_{\left(X_{0}, Y_{0}\right)}=\bigcup_{\theta \in \mathscr{Q}} \mathbf{H}_{\theta}\left(X_{0}, Y_{0}\right)$ where $\mathscr{Q} \subseteq\{1,2, \ldots, n\}$ and $\left|V\left(D_{\theta}\right)\right|>1$ for $\theta \in \mathscr{Q}$.

Let $\mathbf{F}_{M_{\emptyset}}$ be the family of covers of $M_{\emptyset}$ in $\mathscr{B}(G, M, I)$. Considering Lemma 2 and Section 2, for $(a, b) \in \mathbf{F}_{\left(X_{0}, Y_{0}\right)}$ and $\left(X_{1}, Y_{1}\right) \in \mathbf{F}_{M_{\emptyset}} \backslash\left(X_{0}, Y_{0}\right),(a, b)$ will not compare with $\left(X_{1}, Y_{1}\right)$. Therefore, we could say that when $\mathbf{F}_{\left(X_{0}, Y_{0}\right)}$ is carried out, simultaneously, the relation between $(a, b)$ and $(c, d)$ are represented, besides, $h(a, b)=2$, where $(c, d) \in \mathbf{F}_{M_{\emptyset}}$ and $h$ is the height function of $\mathscr{B}(G, M, I)$.

Since $\left|\mathbf{F}_{M_{\emptyset}}\right|<\infty$, repeated application of this above process successively for other members in $\mathbf{F}_{M_{\emptyset}}$, as the consequence, we will obtain all the members $\mathbf{F}_{2}$ in $\mathscr{B}(G, M, I)$ with height 2 and the relationships among $(u, v) \in \mathbf{F}_{2}$ and $(e, f) \in \mathbf{F}_{M_{\emptyset}}$.

Finally, by the principle of induction and recursively compute the covers of each element in a breath-first fashion, we will get all the concepts for a given context $(G, M, I)$ and the diagram $\mathscr{B}(G, M, I)$.

By the definition of $D_{I_{A B}}$, for $x \in G \backslash A$, it must have $d_{D_{I_{A B}}}(x) \leq d_{D_{I}}(x) \leq|M|<\infty$. Thus, after finite steps, the above process must be stopped and get $G_{\emptyset}$ as the last obtained element in $\mathscr{B}(G, M, I)$. Namely, it is a practicable approach provided above to get the members in $\mathscr{B}(G, M, I)$ and the diagram of $\mathscr{B}(G, M, I)$.

\section{Example}

We give an example to show how to use the manners presented from Section 2 to Section 5 to find out the concept lattice $\mathscr{B}(G, M, I)$ and its diagram for a given context $(G, M, I)$.

Example Let $M=\{1,2,3,4,5,6\}$ and $G=\{a, b, c, d, e, f\}$. The table below describes binary relation $I$.

Table 1 The binary relation $I$ for the given context

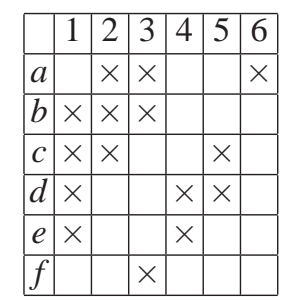

By the following steps to find out $\mathscr{B}(G, M, I)$.

The underlying graph $D_{I}$ associated to $(G, M, I)$ is shown as Figure 1.

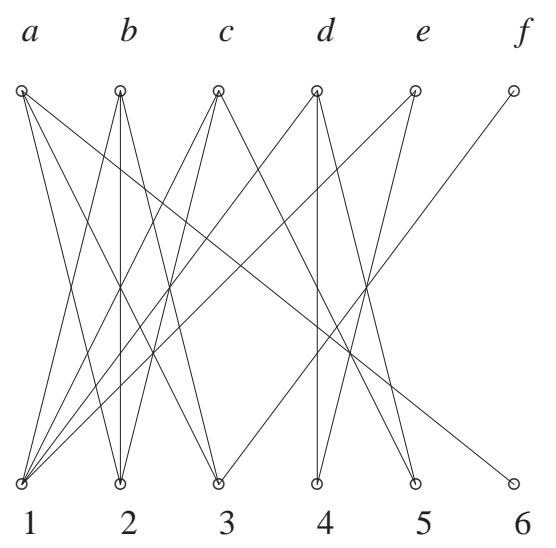

Figure 1 Underlying graph $D_{I}$

Since

$f(f 3) 3(3 b) b(b 1) 1(1 e) e(e 4) 4(4 d) d(d 1) 1(1 c) c(c 2) 2$

$(2 a) a(a 6) 6(6 a) a(a 2) 2(2 b) b(b 1) 1(1 c) c(c 5) 5$ is a $(f, 5)$-path throughout all the vertices in $D_{I}$, where $(x y)$ is the edge $x y$, for $x, y \in V\left(D_{I}\right)$, it follows that $D_{I}$ is connected.

By $D_{I}$, it is easier to get $d(a)=d(b)=d(c)=d(d)=$ $3, N(a)=\{2,3,6\}, N(b)=\{1,2,3\}, N(c)=$ $\{1,2,5\}, N(d)=\{1,4,5\} ; d(e)=2, N(e)=\{1,4\}$; $d(f)=1, N(f)=\{3\}$. Thus, we have $d(f)=1$ $=\min \{d(a), \ldots, d(f)\}<d(e)<d(a)=d(b)=d(c)=$ $d(d)=\max \{d(a), \ldots, d(f)\}$.

Because $N(a) \cap N(g) \neq N(a)$ where $g=b, c, d$. This hints $N(a)^{\prime}=\{a\}$. By the consequence in Section 3, 
$\left(N(a)^{\prime}, N(a)\right)=(\{a\},\{2,3,6\}) \in \mathscr{B}(G, M, I)$ is sound, and simultaneously, it covers $(\emptyset, M)$.

Similarly, $\left(N(b)^{\prime}, N(b)\right)=(\{b\},\{1,2,3\})$,

$\left(N(c)^{\prime}, N(c)\right)=(\{c\},\{1,2,5\})$ and

$\left(N(d)^{\prime}, N(d)\right)=(\{d\},\{1,4,5\})$, besides, all of the three cover $(\emptyset, M)$.

Because $\{x \in G \mid d(x)=2\}=\{e\}$, and additionally, $N(e) \subset N(d)$ hints $\{x \in G \mid d(x)=2$, there is $g \in G$ satisfying $N(x) \subset N(g)\}=\{e\}$. Hence, $\mathscr{T}_{2}=\emptyset$. According to $\{x \in G \mid d(x)=1\}=\{f\}$ and $N(f) \subset N(d)$ hints $\{x \in G \mid d(x)=2$, there is $g \in G$ satisfying $N(x) \subset N(g)\}=\{f\}$. Thus $\mathscr{T}_{1}=\emptyset$.

Therefore, all the cover elements of $(\emptyset, M)$ is $\mathbf{F}_{(\emptyset, M)}=\left\{\left(N(a)^{\prime}, N(a)\right),\left(N(b)^{\prime}, N(b)\right),\left(N(c)^{\prime}, N(c)\right)\right.$, $\left.\left(N(d)^{\prime}, N(d)\right)\right\}=\{(\{a\},\{2,3,6\}),(\{b\},\{1,2,3\})$, $(\{c\},\{1,2,5\}),(\{d\},\{1,4,5\})\}$.

We see $h\left(N(a)^{\prime}, N(a)\right)=1$. A new context associated with $\left(N(a)^{\prime}, N(a)\right)$ is $\left(G \backslash N(a)^{\prime}, N(a), I_{N(a)^{\prime} N(a)}\right)=$ $\left(\{b, c, d, e, f\},\{2,3,6\}, I_{\{a\}\{2,3,6\}}\right)$. In $\mathscr{B}\left(\{b, c, d, e, f\},\{2,3,6\}, I_{\{a\}\{2,3,6\}}\right)$, the minimum is $(\emptyset,\{2,3,6\})$

The underlying graph $D_{I_{\{a\}\{2,3,6\}}}$ is as Figure 2 .

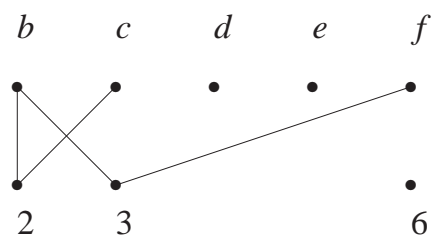

Figure 2 Underlying graph $D_{I_{\{a\}\{2,3,6\}}}$

We find that $D_{I_{\{a\}\{2,3,6\}}}$ has four components $D\left[V_{1}\right]=D(\{b, c, f\} \cup\{2,3\}), D[d], D[e]$ and $D[6]$. According to Lemma 4 and the other results in Section 5, it only needs to consider $D\left[V_{1}\right]$ to search out the covers of $(\{a\},\{2,3,6\})$.

Because $N_{D\left[V_{1}\right]}(b)=\{2,3\}=V\left(D\left[V_{1}\right]\right) \cap M$, considering Status 3 in Section 2 with the discussion in Section 5 , we obtain that $(\{b\},\{2,3\})$ is the minimum in $\mathscr{B}\left(\{b, c, f\},\{2,3\}, I_{1}\right)$, where $(X, Y) \in I_{1} \Longleftrightarrow(X, Y) \in I_{\{a\}\{2,3,6\}}$ for any $(X, Y) \subseteq\{b, c, f\} \times\{2,3\}$.

Moreover, by Theorem $5,(\{b\} \cup\{a\},\{2,3\})=$ $(\{a, b\},\{2,3\})$ is a cover of $(\{a\},\{2,3,6\})$ in $\mathscr{B}(G, M, I)$. Therefore, by the minimum property of $(\{b\},\{2,3\})$ in $\mathscr{B}\left(\{b, c, f\},\{2,3\}, I_{1}\right)$, one gets that in $\mathscr{B}(G, M, I)$, the set of cover elements of $(\{a\},\{2,3,6\})$ is consisted by only $(\{b\} \cup\{a\},\{2,3\})=(\{a, b\},\{2,3\})$.

For any of the other cover elements of a member in $\mathbf{F}_{(\emptyset, M)} \backslash(\{a\},\{2,3,6\})$, by the similar way as the above, we will get their covers. That is to say, we get $\mathbf{F}_{2}$, all the members in $(G, M, I)$ with height 2 , and the relationships between $\mathbf{F}_{2}$ and $\mathbf{F}_{(\emptyset, M)}$. The diagram of $\mathbf{F}_{2} \cup \mathbf{F}_{(\emptyset, M)} \cup(\emptyset, M)$ in $\mathscr{B}(G, M, I)$ is shown as Figure 3 .

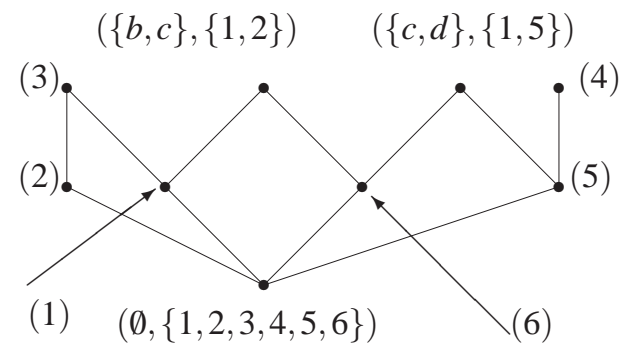

Figure 3 Diagram of $\mathbf{F}_{2} \cup \mathbf{F}_{(\emptyset, M)} \cup(\emptyset, M)$ in the concept lattice

where $(1)=(\{b\},\{1,2,3\}),(2)=(\{a\},\{2,3,6\})$,

(3) $=(\{a, b\},\{2,3\}),(4)=(\{d, e\},\{1,4\})$

$(5)=(\{d\},\{1,4,5\}),(6)=(\{c\},\{1,2,5\})$

Analogously, we will obtain all the members in $\mathscr{B}(G, M, I)$ and at the last, the diagram of $\mathscr{B}(G, M, I)$ is produced at the same time. The diagram of $\mathscr{B}(G, M, I)$ is shown as Figure 4.

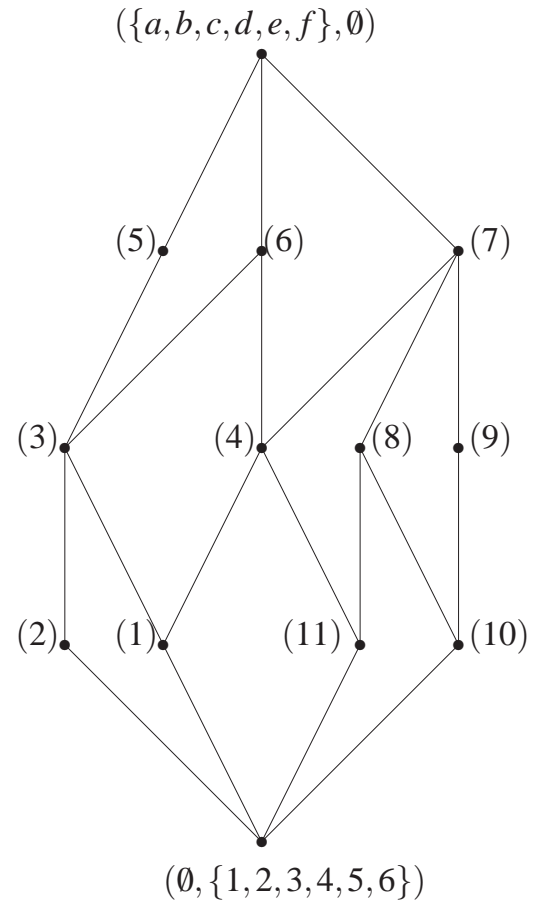

Figure 4 Diagram of the concept lattice

where $(1)=(\{b\},\{1,2,3\}),(2)=(\{a\},\{2,3,6\})$, $(3)=(\{a, b\},\{2,3\}),(4)=(\{b, c\},\{1,2\})$, $(5)=(\{a, b, f\},\{3\}),(6)=(\{a, b, c\},\{2\})$, $(7)=(\{b, c, d, e\},\{1\}),(8)=(\{c, d\},\{1,5\})$, $(9)=(\{d, e\},\{1,4\}),(10)=(\{d\},\{1,4,5\})$, $(11)=(\{c\},\{1,2,5\})$.

At the final part, we say that this Example above is just the running example in [4]. [4] solves this example by its way which is quite different from ours born in this 
paper. Here, using the approach provided in this paper, we obtain the same result about the running example as that in [4]. This also illustrates that the approach here is an efficient computing method and would be a better generating algorithm for concepts.

\section{Acknowledgement}

The author is grateful to the anonymous referee for a careful checking of the details and for helpful comments that improved this paper. This research is supported by NSF of China $(11101115,61073121)$ and NSF of Hebei Province (F2012402037, A2013201119).

\section{References}

[1] B. Ganter and R. Wille, In: General Lattice Theory, 2nd. ed., G.Grätzer (Eds.) (Birkhäuser Verlag, Basel, 1998).

[2] J. A. Bondy and U. S. R.Murty, Graph Theory with Applications (Elsevier Science Publishing Co. Inc., New York, 1976).

[3] G. Grätzer, General Lattice Theory, 2nd. ed. (Birkhäuser Verlag, Basel, 1998).

[4] Anne Berry and Alain Sigayret, Discrete Applied Mathematics, 144, (2004).

[5] J. Abello, A. J. Pogel and L. Miller, J. of Universal Computer Science, 10, 8 (2004).

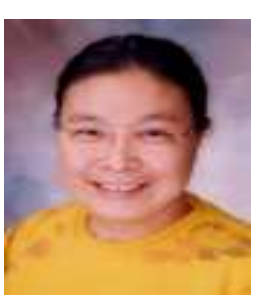

Hua Mao obtained $\mathrm{PhD}$ at Xidian University in 2002 and was a postdoctoral fellow at Department of Mathematics in Zhengzhou University from 2002 to 2004. Since then she is a professor at Department of Mathematics in Hebei University. 